\title{
Second malignancies after breast cancer: The impact of adjuvant therapy (Review)
}

\author{
CHUNHUI DONG and LING CHEN \\ Department of Oncology, The First Affiliated Hospital, College of Medicine, \\ Xi'an Jiaotong University, Xi'an, Shaanxi 710061, P.R. China
}

Received August 9, 2013; Accepted December 2, 2013

DOI: $10.3892 / \mathrm{mco} .2014 .250$

\begin{abstract}
Second malignant neoplasms (SMNs) are potentially life-threatening late sequelae of the adjuvant therapy for breast cancer (BC). The increased risk of SMNs is associated with adjuvant chemotherapy (development of secondary acute myeloid leukemia and myelodysplastic syndrome) and hormonal therapy (risk of uterine cancer secondary to tamoxifen treatment). Previous studies have demonstrated an increased risk of SMNs associated with alkylating agents, topoisomerase-II inhibitors, granulocyte-stimulating factors and estrogen receptor modulators. Furthermore, analytical investigations have demonstrated that $\mathrm{BC}$ patients may be at an increased risk of leukemia following chemotherapy. In addition, correlations between an increased dose of hormonal therapy and solid tumor risk have been identified. Considering the ongoing alterations in the treatment of $\mathrm{BC}$, with respect to lowering the daily as well as the cumulative dose of chemotherapeutic agents, it is anticipated that leukemias will have a considerably lower impact on BC survivors in the future. However, diligent follow-up is required to accurately evaluate the long-term risks associated with chemotherapy.
\end{abstract}

\section{Contents}

1. Introduction

2. Overview of adjuvant therapy

3. Second malignant neoplasms

4. Conclusion

Correspondence to: Professor Ling Chen, Department of Oncology, The First Affiliated Hospital, College of Medicine, Xi'an Jiaotong University, 277 Yanta West Road, Xi'an, Shaanxi 710061, P.R. China

E-mail: cling_12345@163.com

Key words: breast cancer, adjuvant chemotherapy, adjuvant endocrine therapy, human epidermal growth factor receptor 2 directed therapies, second malignant neoplasms

\section{Introduction}

Breast cancer $(\mathrm{BC})$ is one of the most frequently diagnosed types of cancer among females, accounting for $23 \%$ of the total cancer cases (1). Despite its high incidence, the mortality rate of $\mathrm{BC}$ is low (15\%), with a growing number of long-term survivors due to early diagnosis and the increasing use of adjuvant therapy $(2,3)$. The current relative survival rates for all types of BC combined was reported to be $88.8 \%$ at 5 years (79.5\% at 10 years) for Caucasian females, but only $75.3 \%$ at 5 years (63.9\% at 10 years) for females of African descent (4). Second malignant neoplasms (SMNs) are one of the most serious and life-threatening late adverse effects experienced by the growing number of BC survivors worldwide. The risk of SMNs is associated with adjuvant therapy, including chemotherapy, which has been particularly linked to the development of secondary acute myeloid leukemia (AML) and myelodysplastic syndrome (MDS), and hormonal therapy, with an increased risk of uterine cancer secondary to tamoxifen treatment. A previous study reported that the overall risk of developing second malignancies was increased by $18 \%$ among 322,863 females diagnosed with primary BC between 1973 and 2000 [observed/expected $(\mathrm{O} / \mathrm{E})=1.18 ; \mathrm{O}=34,500$; excessive absolute risk $=23$ per 10,000 person-years] (4).

\section{Overview of adjuvant therapy}

The use of adjuvant therapy for BC has evolved, due to extensive investigations and clinical research that have contributed to the understanding of the complexity of breast tumors. Adjuvant systemic therapies include chemotherapy, endocrine therapy and human epidermal growth factor receptor 2 (HER2)-directed therapies.

Chemotherapy. Adjuvant chemotherapy has been applied to eradicate local or distant residual microscopic metastatic disease, with potentially curative effects. By the year 2000, the National Institutes of Health Consensus Development Conference on adjuvant therapy for $\mathrm{BC}$ recommended that adjuvant chemotherapy be considered for nearly all patients with tumors $\geq 1 \mathrm{~cm}$ (5). Indeed, adjuvant chemotherapy has been routinely used in oncology practice for almost all patients, with the exception of those with small, node-negative and well-differentiated cancers (6). 
Over the past few decades, adjuvant chemotherapy for BC has undergone major changes, expanding from the classical cyclophosphamide, methotrexate and 5-fluorouracil (CMF) regimen used in the 1970 s, to anthracycline-containing regimens in the $1990 \mathrm{~s}$, to the recent incorporation of taxanes (paclitaxel and docetaxel) into anthracycline-based regimens (7-10) for the treatment of node-positive or even lower-risk patients. In 1976, Bonadonna et al (11) reported the efficacy of CMF as an adjuvant treatment for patients with node-positive BC. In the mid-1980s, anthracyclines were included in clinical trials. During the 1990s, the administration of 6 cycles of a three-drug anthracycline-containing combination became the standard of care in adjuvant chemotherapy $(12,13)$. The antineoplastic activity of taxanes appears to be associated with their ability to promote microtubular assembly and inhibit microtubular disassembly. In the early 1990s, taxanes (paclitaxel and docetaxel) exhibited potent antitumor efficacy in advanced BC and received approval for inclusion in adjuvant chemotherapy trials. Taxane- and anthracycline-containing regimens are currently the standard adjuvant therapies used for lymph node-positive and possibly high-risk lymph node-negative $\mathrm{BC}$ patients. An increasing number of females receiving adjuvant chemotherapy for $\mathrm{BC}$ also receive granulocyte-stimulating factors to reduce the myelosuppressive effects of dose-intense chemotherapy.

Endocrine therapy. Adjuvant hormone therapy for BC emerged following the identification of the estrogen receptor (ER) in the 1960s and is currently considered a standard treatment for all patients with endocrine-sensitive tumors, as determined by the expression of ER and progesterone receptor by immunohistochemistry. Tamoxifen has been the drug of choice for several years and is used in pre- and postmenopausal females with BC (14). However, in premenopausal females, tamoxifen remains the only endocrine agent approved by the Food and Drug Administration (FDA) for use in the adjuvant setting. The effectiveness of tamoxifen, a selective estrogen receptor modulator (SERM), in blocking the growth of ER-positive cancer cells was demonstrated by previous clinical trials $(15,16)$, with 5 years of tamoxifen being considered the gold standard of hormonal therapy for $\mathrm{BC}$ over the last 30 years.

Furthermore, anastrozole was approved in 1996 for the treatment of metastatic endocrine-sensitive BC. Aromatase inhibitors (AIs) are not used in premenopausal females, as oestrogen is produced in the ovaries until menopause and by the adrenal glands after menopause; as AIs target the adrenal gland-produced oestrogens, their use premenopausally would be of no benefit. Current guidelines recommend incorporating AIs either as primary (initial) therapy, as sequential treatment (after 2-3 years of tamoxifen) or in the extended adjuvent setting (after 5 years of tamoxifen) in postmenopausal women with HR-positive breast cancer (17). If a female patient becomes postmenopausal during the treatment, it may be necessary to consider extended adjuvant therapy with an AI for an additional 5 years.

HER2-directed therapies. Currently FDA-approved HER2-targeted agents, including trastuzumab, lapatinib, pertuzumab and trastuzumab-emtansine (T-DM1), to be refractory. Trastuzumab is a humanized monoclonal antibody directed against the extracellular domain of the HER 2 receptor, which prevents ligand-independent HER2 signaling and was introduced in the treatment of HER2-positive metastatic BC following approval by the FDA in 1998 (18).

Lapatinib is a dual epidermal growth factor receptor/ErbB2 reversible tyrosine kinase inhibitor (blocking HER1 and HER2), which suppresses the downstream signaling of the mitogen-activated protein kinase/extracellular signal-regulated kinase $1 / 2$ and the phosphatidylinositol 3-kinase/Akt pathways. Lapatinib was approved for BC based on a previous study published by Geyer et al (19), which demonstrated that lapatinib plus capecitabine was superior to capecitabine alone for the treatment of females with HER2-positive advanced BC who exhibited disease progression following treatment with regimens that included trastuzumab and chemotherapeutic agents.

Pertuzumab is a monoclonal antibody, which blocks the heterodimerization of HER2 with HER3 by interfering with the ligand-dependent HER3-mediated signaling (20).

T-DM1, which was approved in February, 2013 is an immunoconjugate agent combining trastuzumab with an antimicrotubule cytotoxic chemotherapeutic agent linked by a covalent bond.

Novel HER2-directed agents include pazopanib, afatinib and neratinib. Pazopanib is a selective multitargeted receptor tyrosine kinase inhibitor of vascular endothelial growth factor receptors 1,2 and 3 , platelet-derived growth factor receptor $\alpha / \beta$ and cytokine receptor c-kit, which blocks tumor growth and inhibits angiogenesis. Afatinib and neratinib are irreversible binders of the HER receptors (HER1, HER2 and HER3) (20).

\section{Second malignant neoplasms}

\section{Risks following chemotherapy.}

Leukemia and MDS. Patt et al (21) reported that the absolute risk of developing AML 10 years after any adjuvant chemotherapy for BC was 1.8 vs. $1.2 \%$ for females who had not received chemotherapy. The adjusted hazard ratio (HR) for AML with adjuvant chemotherapy vs. no chemotherapy was 1.53 [95\% confidence interval (CI): 1.14-2.06]. The association of alkylating chemotherapy and topoisomerase-II inhibitor-based chemotherapy with the risk of developing acute leukemia have been well-established $(22,23)$.

Melphalan-based chemotherapy, which was mainly used in the 1970s, is known to be highly leukemogenic (24). The risk of developing therapy-related myeloid neoplasms in patients treated with melphalan was reported to be higher by 10 -fold compared with that of patients who received cyclophosphamide (24). Fisher et al (25) reported that the 10-year cumulative risk of AML was increased in patients treated with surgery followed by melphalan-based chemotherapy, compared to those treated with surgery alone ( 1.29 vs. $0.27 \%$, respectively).

The issue of the leukemogenic potential of cyclophosphamide emerged in the following years. Only a negligible or small increase in risk was reported following a standard dose of CMF chemotherapy $(7,24)$. However, a highly significant correlation between the increasing cumulative dose of cyclophosphamide and the risk of leukemia was reported. The risk for developing AML/MDS in patients with early-stage BC who had been treated with adjuvant chemotherapy containing 
standard dose cyclophosphamide was shown to be higher compared to that in the general population $(7,26)$.

AML associated with alkylating agents, often classified as M1 or M2 with abnormalities in chromosomes 5 and 7, typically develops 5 years after the initial treatment and has a poor prognosis. By contrast, AML associated with topoisomerase-II inhibitors commonly develops within 5 years of therapy and is frequently associated with 11q23 cytogenetic abnormality (27).

Cyclophosphamide-anthracycline-based regimens, which have been widely used over the last few years, may be associated with a higher risk of leukemia; however, in absolute terms, the risk appears low when standard doses are administered (cumulative incidence of $<0.5 \%$ at $8-10$ years) (28). The French Adjuvant Study Group reported that, irrespective of the dose, the incidence of secondary leukemia following adjuvant epirubicin-based chemotherapy was low (8). After 9 years, the risk of developing leukemia was $0.34 \%$ (95\% CI: 0.11-0.57) in epirubicin-exposed patients. Furthermore, Smith et al (28) performed a combined analysis of six adjuvant studies conducted by the National Surgical Adjuvant Breast and Bowel Project group, using regimens containing doxorubicin and cyclophosphamide. That study reported a 5-year incidence of AML of $0.3-1.2 \%$, with an increased risk for greater dose intensity. However, at least two reports from the clinical trial series indicated that the risk of leukemia may rise sharply with more intensive, higher-dose chemotherapy regimens (28-30), indicating that these patients must be closely monitored for the late effects of therapy. In addition, patients treated with standard cumulative doses of adjuvant epirubicin $\left(\leq 720 \mathrm{mg} / \mathrm{m}^{2}\right)$ and cyclophosphamide $\left(\leq 6,300 \mathrm{mg} / \mathrm{m}^{2}\right)$ for early $\mathrm{BC}$ have a lower probability of secondary leukemia compared to patients treated with higher cumulative doses (29). In order to assess the risk of developing AML and MDS following exposure to epirubicin-based regimens, Praga et al (29) reviewed a total of 7,110 patients treated with epirubicin and cyclophosphamide in 19 randomized clinical trials in 2005. Patients who were administered cumulative doses of epirubicin and cyclophosphamide not exceeding those used in standard regimens ( $\leq 720$ and $\leq 6,300 \mathrm{mg} / \mathrm{m}^{2}$, respectively) had an 8 -year cumulative probability of developing AML/MDS of $0.37 \%$ (95\% CI: 0.13-0.61) compared to $4.97 \%$ (95\% CI: 2.06-7.87) for patients who received higher cumulative doses of epirubicin and cyclophosphamide. The significance of dose intensity was also confirmed with the 'intense dose-dense' regimen comprising epirubicin, paclitaxel and cyclophosphamide every 2 weeks. This regimen proved more effective compared to the standard regimen of epirubicin/cyclophosphamide and improved event-free and overall survival; however, it was also more toxic, leading to four cases $(0.6 \%$ of the patients) with therapy-related AML/MDS (30). Therefore, the increased risk of secondary leukemia must be considered when assessing the potential benefits of higher compared to standard doses.

A dose-dependent increase in the risk of leukemia was observed in females treated with mitoxantrone (31). A large case-control study demonstrated that the risk of AML/MDS was increased following topoisomerase-II inhibitor-based chemotherapy, with the risk of leukemia being higher for mitoxantrone-based compared to anthracycline-based chemotherapy (23). The Cox regression analysis revealed that the risk of leukemia was significantly lower in patients treated with anthracyclines compared to that in patients treated with mitoxantrone at cumulative doses of $>13 \mathrm{mg} / \mathrm{m}^{2}$ (31).

As regards taxanes, a Surveillance, Epidemiology and End Results (SEER) database analysis (21) and data from large adjuvant trials did not demonstrate an increased risk of leukemia following the administration of paclitaxel or docetaxel (32-34). A 7-year follow-up of a trial comparing doxorubicin/cyclophosphamide (AC) with docetaxel/cyclophosphamide (TC) treatment in patients with early $\mathrm{BC}$, reported no secondary leukemia in the TC arm, compared to 2 cases among 510 patients $(0.4 \%)$ in the AC arm (32). A three-arm study comparing the Canadian CEF regimen (cyclophosphamide $75 \mathrm{mg} / \mathrm{m}^{2}$, days 1-14; epirubicin $60 \mathrm{mg} / \mathrm{m}^{2}$, days 1 and 8 ; and 4-fluorouracil $500 \mathrm{mg} / \mathrm{m}^{2}$, days 1 and 8) to 2-weekly dose-dense EC (epirubicin $120 \mathrm{mg} / \mathrm{m}^{2}$, day 1; and cyclophosphamide $830 \mathrm{mg} / \mathrm{m}^{2}$, day 1) followed by paclitaxel $\left(175 \mathrm{mg} / \mathrm{m}^{2}\right.$, day 1) and to 3-weekly AC followed by paclitaxel, demonstrated that $0.5 \%$ of the patients in the first two arms developed AML (34), which is mainly attributed to the high cumulative dose of epirubicin. However, no secondary leukemia was diagnosed in patients randomly assigned to the $\mathrm{AC} /$ paclitaxel arm.

The leukemogenic effect of granulocyte colony-stimulating factor (G-CSF) remains a subject of considerable controversy. An analysis of the SEER-medicare population-based database, including 5,510 females with $\mathrm{BC}$ treated with adjuvant chemotherapy, indicated that the addition of G-CSF was associated with a doubling of the risk of subsequent AML or MDS when compared to chemotherapy alone, even if the absolute risk remained low (35). Similarly, in another study, patients receiving G-CSF support exhibited an increased risk of AML/MDS (relative risk $=6.3$; 95\% CI: 1.9-21), even when controlling for chemotherapeutic doses (23). By contrast, Patt et al (21) did not identify an increased risk of AML in elderly ( $>65$ years) BC patients who received G-CSF in the first years following diagnosis as part of the adjuvant therapy. In the Cancer and Leukemia Group B 9741 phase III trial, the patients received dose-dense regimens plus filgrastim support, but exhibited no increased risk of developing AML or MDS compared to those treated with the same conventional regimen without G-CSF (36). More recently, treatment with rituximab as high-dose therapy with autologous stem-cell transplantation for lymphoma has been implicated as a possible risk factor for the subsequent development of solid tumors (37).

\section{Risks following hormonal therapy.}

Solid cancers. There is increasing evidence that adjuvant treatment affects the risk of developing contralateral breast cancer (CBC). However, adjuvant hormonal therapy was found to considerably reduce the risk of $\operatorname{CBC}(15,38,39)$. A meta-analysis by the Early Breast Cancer Trialists' Collaborative Group reported that tamoxifen administration for 2 or 5 years resulted in proportional reductions of the incidence of CBC of 26 and 47\%, respectively (15). Furthermore, a population-based study demonstrated that adjuvant hormonal therapy [HR=0.58; 95\% CI: 0.48-0.69] was associated with a markedly decreased CBC risk (40). Adjuvant hormonal therapy reduces the risk of $\mathrm{CBC}$ in $\mathrm{BC}$ survivors, but preliminary data indicated that it may also increase the risk of hormone receptor-negative contralateral tumors. A population-based nested case-control study indicated that, 
compared with females not treated with hormonal therapy, users of adjuvant tamoxifen for $\geq 5$ years exhibited a reduced risk of $\mathrm{ER}+\mathrm{CBC}$ [odds ratio $(\mathrm{OR})=0.4,95 \% \mathrm{CI}$ : 0.3-0.7], but also exhibited a 4.4-fold (95\% CI: .03-19.0) increased risk of ER-CBC (41). Furthermore, that study reported a significant time-dependent phenomenon, that tamoxifen use for $<5$ years was not associated with ER-CBC risk. Recent data suggested that AIs may be even more effective in reducing CBC (42-44).

Various studies revealed that tamoxifen causes endometrial cancer $(15,38,39,43)$, with a significantly higher risk for tamoxifen-related uterine sarcomas, with a positive correlation between risk and increased duration of tamoxifen use (39,45-47). In a case-control study, tamoxifen treatment, compared to no treatment, was associated with an increased risk of endometrial cancer $(\mathrm{OR}=2.4 ; 95 \% \mathrm{CI}$ : 1.8-3.0). The risk increased significantly $(\mathrm{P}<0.001)$ with the duration of treatment (for $\geq 5$ years of treatment compared to no treatment, $\mathrm{OR}=3.6$, 95\% CI: 2.6-4.8) (39). Curtis et al (45) evaluated data from 39,451 patients diagnosed with BC between 1980 and 2000, who were initially treated with tamoxifen, and observed that the overall risk of subsequent uterine corpus cancer was increased by $>2$-fold (O/E 2.17, 95\% CI: 1.95-2.41) compared to the general SEER population. Similarly, using Cancer Registry data, Kamigaki and Kawakami (48) revealed that the incidence rate ratio of corpus uteri cancer associated with hormonal therapy was 2.53 (95\% CI: 1.41-4.55). Yadav et al (49) found that there was a significantly higher incidence of endometrial and ovarian cancer in patients treated with adjuvant hormonal therapy (5.2\%) compared to patients not administered hormonal therapy $(1.8 \%, \mathrm{P}=0.002)$. The risk of endometrial cancer adjusted for treatment duration did not diminish in $\geq 5$ years after the last treatment ended (39). The risk of endometrial cancer was not associated with the daily dose of tamoxifen and was comparable in pre- and postmenopausal females (39). The increased risk was found to occur predominantly among females aged $\geq 50$ years (38) and treatment duration may affect the magnitude of the risk (50).

Other SERMs, such as raloxifene and toremifene, may be safer for the uterus and a lower risk of endometrial cancer was reported (50-53). At the first interim analysis on 899 out of 1,489 accrued patients following a median follow-up of 3.4 years, two cases of endometrial cancer were observed in the tamoxifen arm and none in the toremifene arm (50). By contrast, the first International Breast Cancer Study Group (IBCSG) study comparing toremifene and tamoxifen (IBCSG Trials 12-93 and 14-93) as adjuvant hormonal treatments for early BC, reported a similar incidence of second primary cancer with the two agents after a median follow-up of 5.5 years, with no significant difference in the incidence of endometrial cancer (54).

Previous studies indicated that the tamoxifen-related risk of uterine corpus cancer may be particularly high for certain uncommon cell types, although the magnitude of risk has not been determined $(39,45)$. Treatment with tamoxifen was associated with a significantly higher risk of Müllerian and mesodermal-mixed endometrial tumors $(\mathrm{OR}=13.5$, 95\% CI: 4.1-44.5) compared to that of adenocarcinoma $(\mathrm{OR}=2.1,95 \% \mathrm{CI}: 1.6-2.7)$ or clear cell and papillary serous tumors (OR=3.1, 95\% CI: 0.8-17.9) (39). In another study using data from nine population-based cancer registries in the SEER Program, the relative risk was substantially higher for malignant mixed Müllerian tumors (MMMTs) $(\mathrm{O} / \mathrm{E}=4.62$, $\mathrm{O}=34,95 \% 95 \% \mathrm{CI}: 3.20-6.46)$ compared to that for endometrial adenocarcinomas $(\mathrm{O} / \mathrm{E}=2.07, \mathrm{O}=306,95 \% \mathrm{CI}: 1.85-2.32)$, although the excess absolute risk was smaller, an additional 1.4 vs. 8.4 cancers per 10,000 females per year, respectively (45). Among those who survived for $\geq 5$ years, there was an 8-fold relative risk for MMMTs and a 2.3-fold risk for endometrial adenocarcinomas, with patients who developed MMMTs exhibiting a worse prognosis (45).

Patients with endometrial cancer secondary to $\mathrm{BC}$ who received tamoxifen treatment for $\mathrm{BC}$ for 5 years, exhibited a higher endometrial cancer mortality risk compared to those who did not receive tamoxifen ( $\mathrm{HR}=1.59,95 \% \mathrm{CI}$ : 1.13-2.25) (53). This may be attributed to the non-endometrioid histological subtypes with a poorer prognosis among long-term tamoxifen users $(39,45,55)$. However, there have been studies which indicated that the incidence of endometrial abnormalities and their clinicopathological characteristics were independent of tamoxifen use (56). In a recent retrospective study that investigated whether the incidence of endometrial abnormalities and their clinicopathological characteristics were affected by receiving tamoxifen, non-steroidal AIs or no treatment, Le Donne et al (56) reported cancer in $3.8 \%$ of the cases in the tamoxifen group and $11.1 \%$ of the cases in the no treatment group. Slomovitz et al (57) reported that females who developed endometrial cancer following a diagnosis of $\mathrm{BC}$ were at an increased risk of developing the high-risk histological subtypes, independent of tamoxifen use. No significant differences were identified in the clinical or pathological characteristics of endometrial cancer between tamoxifen users and non-users. Fles et al (58) demonstrated that tumors (all subtypes), which had developed following prolonged use of tamoxifen, were not distinguishable from those of non-users on the basis of their genomic profile.

Antiestrogen therapy was shown to modify the risk of melanoma after BC (59). Compared with the general population, the risk of melanoma was higher for patients who did not receive antiestrogens [standardized incidence ratio (SIR): 1.60, 95\% CI: $1.08-2.12, \mathrm{P}=0.02]$. By contrast, the risk was close to 1 ( $\mathrm{SIR}=0.98,95 \% \mathrm{CI}: 0.40-1.56, \mathrm{P}=0.57)$ for patients who received antiestrogen therapy (59).

HER2-directed therapies have significantly altered the prognosis of HER2-positive breast cancers. However, due to the limited time they have been used and since they are not fully utilized in the clinical setting, there is currently no available study reporting an increased risk of second primary malignancies among BC patients treated with HER2 directed therapies.

\section{Conclusion}

The improvements regarding the early detection of $\mathrm{BC}$ and adjuvant therapy have contributed to the increased number of BC survivors. However, an increased risk of subsequent malignancies following treatment with alkylating agents and topoisomerase-II inhibitors among cancer survivors is well-established, with chemotherapy being linked particularly to the development of secondary AML and MDS and hormonal therapy to the risk of uterine cancer secondary to tamoxifen. Although the absolute risk is low, careful documentation of the magnitude and temporal pattern of these site-specific excess 
risk, as well as delineation of the contribution of treatment exposure and other factors, may facilitate the development of optimal follow-up plans. We should consider that the benefits associated with a number of cancer treatments significantly outweigh the risk of adverse effects. Furthermore, the untoward sequelae of cancer and its therapy may reflect not only the effects of treatment, but also the effect of shared etiological factors, environmental exposure, host characteristics, patient comorbidities, underlying hepatic and renal function, lifestyle factors and the combination of these effects.

\section{References}

1. Jemal A, Bray F, Center MM, Ferlay J, Ward E and Forman D: Global cancer statistics. CA Cancer J Clin 61: 69-90, 2011.

2. DeSantis C, Siegel R, Bandi P and Jemal A: Breast cancer statistics, 2011. CA Cancer J Clin 61: 409-418, 2011.

3. Siegel R, Ward E, Brawley O and Jemal A: Cancer statistics, 2011: the impact of eliminating socioeconomic and racial disparities on premature cancer deaths. CA Cancer J Clin 61: 212-236, 2011

4. Curtis RE, Freedman DM, Ron E, Ries LAG, Hacker DG, Edwards BK, Tucker MA and Fraumeni JF Jr (eds): New malignancies following breast cancer. In: New Malignancies Among Cancer Survivors: SEER Cancer Registries, 1973-2000. National Cancer Institute, NIH Publishing, Bethesda, MD, pp181-205, 2006

5. Eifel P, Axelson J, Costa J, et al: National Institutes of Health Consensus Development Conference Statement: adjuvant therapy for breast cancer, November 1-3, 2000. J Natl Cancer Inst 93: 979-989, 2001.

6. Carlson RW, Allred DC, Anderson BO, et al: Invasive breast cancer. J Natl Compr Canc Netw 9: 136-222, 2011.

7. Tallman M, Gray R, Bennett J, et al: Leukemogenic potential of adjuvant chemotherapy for early-stage breast cancer: the Eastern Cooperative Oncology Group experience. J Clin Oncol 13: $1557-1563,1995$

8. Campone M, Roche H, Kerbrat P, et al: Secondary leukemia after epirubicin-based adjuvant chemotherapy in operable breast cancer patients: 16 years experience of the French Adjuvant Study Group. Ann Oncol 16: 1343-1351, 2005.

9. Renella R, Verkooijen HM, Fioretta G, et al: Increased risk of acute myeloid leukaemia after treatment for breast cancer. Breast 15: 614-619, 2006.

10. Bernard-Marty C, Mano M, Paesmans M, et al: Second malignancies following adjuvant chemotherapy: 6-year results from a Belgian randomized study comparing cyclophosphamide, methotrexate and 5-fluorouracil (CMF) with an anthracycline-based regimen in adjuvant treatment of node-positive breast cancer patients. Ann Oncol 14: 693-698, 2003.

11. Bonadonna G, Brusamolino E, Valagussa P, et al: Combination chemotherapy as an adjuvant treatment in operable breast cancer. N Engl J Med 294: 405-410, 1976.

12. Martin M, Villar A, Sole-Calvo A, et al; GEICAM Group (Spanish Breast Cancer Research Group): Doxorubicin in combination with fluorouracil and cyclophosphamide (i.v. FAC regimen, day 1,21 ) versus methotrexate in combination with fluorouracil and cyclophosphamide (i.v. CMF regimen, day 1,21) as adjuvant chemotherapy for operable breast cancer: a study by the GEICAM group. Ann Oncol 14: 833-842, 2003.

13. Fumoleau P, Kerbrat P, Romestaing P, et al: Randomized trial comparing six versus three cycles of epirubicin-based adjuvant chemotherapy in premenopausal, node-positive breast cancer patients: 10-year follow-up results of the French Adjuvant Study Group 01 trial. J Clin Oncol 21: 298-305, 2003.

14. Benson JR, Jatoi I, Keisch M,Esteva FJ, Makris A and Jordan VC: Early breast cancer. Lancet 373: 1463-1479, 2009.

15. No authors listed: Tamoxifen for early breast cancer: an overview of the randomised trials. Early Breast Cancer Trialists' Collaborative Group. Lancet 351: 1451-1467, 1998.

16. FisherB,JeongJH,BryantJ,etal:Treatment of lymph-node-negative, oestrogen-receptor-positive breast cancer: long-term findings from National Surgical Adjuvant Breast and Bowel Project randomized clinical trials. Lancet 364: 858-868, 2004.

17. Burstein HJ,Prestrud AA, Seidenfeld J, et al: American Society of Clinical Oncology clinical practice guideline: update on adjuvant endocrine therapy for women with hormone receptor-positive breast cancer. J Clin Oncol 28: 3784-3796, 2010
18. Slamon DJ, Leyland-Jones B, Shak S, et al: Use of chemotherapy plus a monoclonal antibody against HER2 for metastatic breast cancer that overexpresses HER2. N Engl J Med 344: 783-792, 2001.

19. Geyer CE, Forster J, Lindquist D, Chan S, Romieu CG, Pienkowski T, et al: Lapatinib plus capecitabine for HER2-positive advanced breast cancer. N Engl J Med 355: 2733-2743, 2006.

20. Gradishar WJ: HER2 therapy - an abundance of riches. N Engl J Med 366: 176-178, 2012

21. Patt DA, Duan Z, Fang S, Hortobagyi GN and Giordano SH: Acute myeloid leukemia after adjuvant breast cancer therapy in older women: understanding risk. J Clin Oncol 25: 3871-3876, 2007.

22. Leone G, Pagano L, Ben-Yehuda D and Voso MT: Therapy-related leukemia and myelodysplasia: susceptibility and incidence. Haematologica 92: 1389-1398, 2007.

23. Le Deley MC, Suzan F, Cutuli B, et al: Anthracyclines, mitoxantrone, radiotherapy, and granulocyte colony-stimulating factor: risk factors for leukemia and myelodysplastic syndrome after breast cancer. J Clin Oncol 25: 292-300, 2007.

24. Curtis RE, Boice JD Jr, Stovall M, et al: Risk of leukemia after chemotherapy and radiation treatment for breast cancer N Engl J Med 326: 1745-1751, 1992.

25. Fisher B, Rockette H, Fisher ER, Wickerham DL, Redmond C and Brown A: Leukemia in breast cancer patients following adjuvant chemotherapy or postoperative radiation: the NSABP experience. J Clin Oncol 3: 1640-1658, 1985.

26. Valagussa P, Moliterni A, Terenziani M, Zambetti $M$ and Bonadonna G: Second malignancies following CMF-based adjuvant chemotherapy in resectable breast cancer. Ann Oncol 5: 803-808, 1994.

27. Pedersen-Bjergaard J, Pedersen M, Roulston D and Philip P: Different genetic pathways in leukemogenesis for patients presenting with therapy-related myelodysplasia and therapy-related acute myeloid leukemia. Blood 86: 3542-3552, 1995.

28. Smith RE, Bryant J, DeCillis A and Anderson S; National Surgical Adjuvant Breast and Bowel Project Experience: Acute myeloid leukemia and myelodysplastic syndrome after doxorubicin-cyclophosphamide adjuvant therapy for operable breast cancer: the National Surgical Adjuvant Breast and Bowel Project Experience. J Clin Oncol 21: 1195-1204, 2003.

29. Praga C, Bergh J, Bliss J, et al: Risk of acute myeloid leukemia and myelodysplastic syndrome in trials of adjuvant epirubicin for early breast cancer: correlation with doses of epirubicin and cyclophosphamide. J Clin Oncol 23: 4179-4191, 2005.

30. Moebus V, Jackisch C, Lueck HJ, et al: Intense dose-dense sequential chemotherapy with epirubicin, paclitaxel, and cyclophosphamide compared with conventionally scheduled chemotherapy in high-risk primary breast cancer: mature results of an AGO phase III study. J Clin Oncol 28: 2874-2880, 2010.

31. Chaplain G, Milan C, Sgro C, Carli PM and Bonithon-Kopp C: Increased risk of acute leukemia after adjuvant chemotherapy for breast cancer: a population-based study. J Clin Oncol 18: 2836-2842, 2000.

32. Jones S, Holmes FA, O'Shaughnessy J, et al: Docetaxel with cyclophosphamide is associated with an overall survival benefit compared with doxorubicin and cyclophosphamide: 7-year follow-up of US Oncology Research Trial 9735. J Clin Oncol 27: $1177-1183,2009$

33. Roché H, Fumoleau P, Spielmann M, et al: Sequential adjuvant epirubicin-based and docetaxel chemotherapy for node-positive breast cancer patients: the FNCLCC PACS 01 Trial. J Clin Oncol 24: 5664-5671, 2006.

34. Burnell M, Levine MN, Chapman JA, Bramwell V, Gelmon K, Walley B, et al: Cyclophosphamide, epirubicin, and fluorouracil versus dose-dense epirubicin and cyclophosphamide followed by paclitaxel versus doxorubicin and cyclophosphamide followed by paclitaxel in node-positive or high-risk node-negative breast cancer. J Clin Oncol 28: 77-82, 2010.

35. Hershman D, Neugut AI, Jacobson JS, et al: Acute myeloid leukemia or myelodysplastic syndrome following use of granulocyte colony-stimulating factors during breast cancer adjuvant chemotherapy. J Natl Cancer Inst 99: 196-205, 2007.

36. Citron ML, Berry DA, Cirrincione C, et al: Randomized trial of dose-dense versus conventionally scheduled and sequential versus concurrent combination chemotherapy as postoperative adjuvant treatment of node-positive primary breast cancer: first report of Intergroup Trial C9741/Cancer and Leukemia Group B Trial 9741. J Clin Oncol 21: 1431-1439, 2003. 
37. Tarella C, Passera R, Magni M, et al: Risk factors for the development of secondary malignancy after high-dose chemotherapy and autograft, with or without rituximab: a 20 -year retrospective follow-up study in patients with lymphoma. J Clin Oncol 29: 814-824, 2011

38. Mellemkjaer L, Friis S, Olsen JH, et al: Risk of second cancer among women with breast cancer. Int J Cancer 118: 2285-2292, 2006.

39. Swerdlow AJ and Jones ME; British Tamoxifen Second Cancer Study Group: Tamoxifen treatment for breast cancer and risk of endometrial cancer: a case-control study. J Natl Cancer Inst 97 375-384, 2005

40. Schaapveld M, Visser O, Louwman WJ, et al: The impact of adjuvant therapy on contralateral breast cancer risk and the prognostic significance of contralateral breast cancer: a population based study in the Netherlands. Breast Cancer Res Treat 110 189-197, 2008

41. Li CI, Daling JR, Porter PL, Tang MT and Malone KE: Adjuvant hormonal therapy for breast cancer and risk of hormone receptor-specific subtypes of contralateral breast cancer. Cancer Res 69: 6865-6870, 2009.

42. Baum M, Budzar AU, Cuzick J, et al: Anastrozole alone or in combination with tamoxifen versus tamoxifen alone for adjuvant treatment of postmenopausal women with early breast cancer: first results of the ATAC randomised trial. Lancet 359: 2131-2139, 2002.

43. Baum M, Buzdar A, Cuzick J, et al: Anastrozole alone or in combination with tamoxifen versus tamoxifen alone for adjuvant treatment of postmenopausal women with early-stage breast cancer: results of the ATAC (Arimidex, Tamoxifen Alone or in Combination) trial efficacy and safety update analyses Cancer 98: 1802-1810, 2003.

44. Goss PE, Ingle JN, Martino S, et al: A randomized trial of letrozole in postmenopausal women after five years of tamoxifen therapy for early-stage breast cancer. N Engl J Med 349: 1793-1802, 2003.

45. Curtis RE, Freedman DM, Sherman ME and Fraumeni JF Jr: Risk of malignant mixed mullerian tumors after tamoxifen therapy for breast cancer. J Natl Cancer Inst 96: 70-74, 2004

46. Early Breast Cancer Trialists' Collaborative Group (EBCTCG): Effects of chemotherapy and hormonal therapy for early breast cancer on recurrence and 15-year survival: an overview of the randomised trials. Lancet 365: 1687-1717, 2005.

47. Bergman L, Beelen ML, Gallee MP, Hollema H, Benraadt J and van Leeuwen FE: Risk and prognosis of endometrial cancer after tamoxifen for breast cancer. Comprehensive Cancer Centres' ALERT Group. Assessment of Liver and Endometrial cancer Risk following Tamoxifen. Lancet 356: 881-887, 2000.

48. Kamigaki $Y$ and Kawakami K: Risk of second cancer after initial treatment of breast cancer: An Osaka Cancer Registry Database study. Oncol Lett 2: 963-973, 2011.
49. Yadav BS, Sharma SC, Patel FD, Ghoshal S, Kapoor R and Kumar R: Nonbreast second malignancies after treatment of primary breast cancer. Int J Radiat Oncol Biol Phys 73: 1489-1492, 2009

50. Matesich SM and Shapiro CL: Second cancers after breast cancer treatment. Semin Oncol 30: 740-748, 2003

51. Holli k, Valavaara R, Blanco G, et al; Finnish Breast Cancer Group: Safety and efficacy results of a randomized trial comparing adjuvant toremifene and tamoxifen in postmenopausal patients with node-positive breast cancer. J Clin Oncol 18: 3487-3494, 2000.

52. Vogel VG, Costantino JP, Wickerham DL, et al; National Surgical Adjuvant Breast and Bowel Project (NSABP): Effects of tamoxifen vs. raloxifene on the risk of developing invasive breast cancer and other disease outcomes: the NSABP Study of Tamoxifen and Raloxifene (STAR) P-2 trial. JAMA 295: 2727-2741, 2006.

53. Pukkala E, Kyyronen P, Sankila R and Holli K: Tamoxifen and toremifene treatment of breast cancer and risk of subsequent endometrial cancer: a population-based case-control study. Int J Cancer 100: 337-341, 2002.

54. International Breast Cancer Study Group, Pagani O, Gelber S, Price K, Zahrieh D, Gelber R, et al: Toremifene and tamoxifen are equally effective for early-stage breast cancer: first results of International Breast Cancer Study Group Trials 12-93 and 14-93. Ann Oncol 15: 1749-1759, 2004.

55. Jones ME, van Leeuwen FE, Hoogendoorn WE, et al: Endometrial cancer survival after breast cancer in relation to tamoxifen treatment: pooled results from three countries. Breast Cancer Res 14: R91, 2012.

56. Le Donne M, Alibrandi A, Ciancimino L, Azzerboni A, Chiofalo B and Triolo O: Endometrial pathology in breast cancer patients: Effect of different treatments on ultrasonographic, hysteroscopic and histological findings. Oncol Lett 5: 1305-1310, 2013.

57. Slomovitz BM, Sun CC, Ramirez PT, Bodurka DC, Diaz P and Lu KH: Does tamoxifen use affect prognosis in breast cancer patients who develop endometrial cancer? Obstet Gynecol 104: 255-260, 2004

58. Fles R, Hoogendoorn WE, Platteel I, et al: Genomic profile of endometrial tumors depends on morphological subtype, not on tamoxifen exposure. Genes Chromosomes Cancer 49: 699-710, 2010.

59. Huber C, Bouchardy C, Schaffar R, et al: Antiestrogen therapy for breast cancer modifies the risk of subsequent cutaneous melanoma. Cancer Prev Res (Phila) 5: 82-88, 2012. 